\title{
The Energetic Particle Spectrometer HEP onboard the GEOTAIL Spacecraft
}

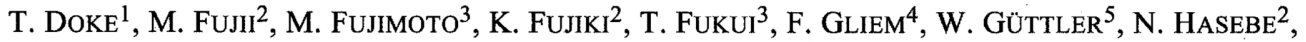

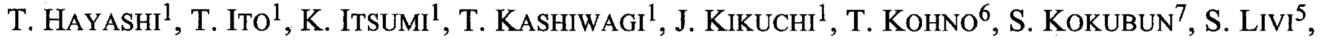 \\ K. Maezawa ${ }^{3}$, H. Moriya ${ }^{2}$, K. Munakata ${ }^{8}$, H. Murakami ${ }^{9}$, Y. Muraki ${ }^{10}$, H. Nagoshi $^{2}$, A. Nakamoto ${ }^{9}$, \\ K. NAGATA ${ }^{11}$, A. NishidA ${ }^{12}$, R. RATHJE ${ }^{5}$, T. SHINO ${ }^{1}$, H. SOMMER $^{5}$, T. TAKASHima ${ }^{1}$, T. TERASAWA ${ }^{13}$,

 \\ ${ }^{1}$ Advanced Research Center for Science and Engineering, Waseda University, Okubo 3-4-1, Shinjuku, Tokyo 169, Japan \\ ${ }^{2}$ Faculty of General Education, Ehime University, Bunkyo, Matsuyama, Ehime 790, Japan \\ ${ }^{3}$ Department of Physics, Nagoya University, Chikusa-ku, Nagoya 464, Japan \\ ${ }^{4}$ Inst. Für Datenverarbeitungsamlagen (IDA), Hans-Sommer-Str. 66, 3300 Braunschweig, FRG \\ ${ }^{5}$ Max-Planck Institut für Aeronomie, 3411 Katlenburg-Lindau, FRG \\ ${ }^{6}$ The Institute of Physical and Chemical Research (RIKEN), Hirasawa, Wako 351-01, Japan \\ ${ }^{7}$ Solar-Terrestrial Environment Laboratory, Nagoya University, Toyokawa 442, Japan \\ ${ }^{8}$ Department of Physics, Shinshu University, Matsumoto, Nagano 390, Japan \\ ${ }^{9}$ Department of Physics, Rikkyo University, Nishi-Ikebukuro, Toshima-ku, Tokyo 171, Japan \\ ${ }^{10}$ Solar-Terrestrial Environment Laboratory, Nagoya University, Chikusa-ku, Nagoya 464, Japan \\ ${ }^{11}$ Faculty of Engineering, Tamagawa University, Machida, Tokyo 194, Japan \\ ${ }^{12}$ The Institute of Space and Astronomical Science, Sagamihara, Kanagawa 229, Japan \\ ${ }^{13}$ Department of Earth and Planetary Physics, University of Tokyo, Bunkyo-ku, Tokyo 113, Japan \\ ${ }^{14}$ Univesitete i Bergen, Fysik Institut, Allegaten 53-55, 5014 Bergen-U, Norway \\ ${ }^{15}$ Department of Earth Science, Ibaraki University, Mito, Ibaraki 310, Japan
}

(Received June 11, 1993; Revised April 4, 1994; Accepted April 11, 1994)

The instruments for the HEP (High Energy Particle) experiments of the GEOTAIL mission, launched in July 1992, consist of 5 spectrometers (LD, BD, MI-1, MI-2 and HI). The LD (Low energy particle Detector) and the BD (Burst Detector) are mainly used to measure electrons, protons, helium and oxygen ions which reflect plasma dynamics in the magnetotail region. On the other hand, MI-1, MI-2 (Medium energy Isotope telescope-1, -2) and HI (High energy Isotope telescope) are used to measure the isotope abundance of solar flare particles and cosmic ray particles which reflect physical conditions of interplanetary space and origin of these particles. In this paper, the objectives of these experiments, the details of the instruments and preliminary results from the observation in the magnetotail are given. The preliminary results obtained by the LD and BD show that a highly collimated beam of energetic particles appear in the plasma sheet just after the substorm onset and that directional distribution of particles can be radically different for different energies. In the $\mathrm{MI}$ and $\mathrm{HI}$ telescopes, details a remarkable enhancement of anomalous cosmic ray components $(\mathrm{N}, \mathrm{O})$ in the quiet time of solar activity is reported, as well as the element composition of galactic cosmic rays.

\section{Introduction}

The HEP (High Energy Particle) subsystem for the GEOTAIL mission has been designed to observe several species of charged particles in a wide energy range to understand the charged particle environment. The objectives of the HEP subsystem can be divided into two categories (Doke et al., 1989, 1990a, 1990b). The first category is the study of plasma dynamics and the second category is the studies of the solar flare mechanism, the anomalous cosmic ray particles and the galactic cosmic ray particles. The HEP instruments consist of 5 different spectrometers (LD, BD, MI-1, MI-2, and HI) and data processing unit (DPU). The LD (Low energy particle Detector), and the BD (Burst Detector) are mainly used to measure electrons, protons, heliums, and ions which reflect plasma dynamics in the magnetotail region. On the other hand, MI-1, MI-2 (Medium energy Isotope telescope-1, -2) and HI (High energy Isotope telescope) 
are mostly sensitive to solar flare particles and cosmic rays which reflect physical conditions of interplanetary space and origin of these particles.

In July, 1992, the GEOTAIL satellite was launched and now in full operation moving around in the scheduled orbit. In this paper, details of the LD and BD spectrometers, the MI and HI telescopes and their electronics system are described as well as their initial results obtained so far.

The following sections describe the principal features of the various HEP sensor systems and associated electronics. Sections 2 and 3 address the detection techniques and specific features of the LD/ $\mathrm{BD}$ spectrometers and the $\mathrm{MI} / \mathrm{HI}$ telescopes. The focus of this paper is to provide an overview of the HEP instrument package and its basic characteristics. This HEP report is complemented by a set of dedicated publications on the LD and the MI/HI spectrometer systems with complete details (Wilken et al., 1994; Doke et al., 1994).

\section{The LD and BD Spectrometers}

The LD/BD complement covers the energetic extension of the plasma distribution function in all plasma regions visited by the GEOTAIL spacecraft. In particular, LD/BD will address the following aspects:

- $\quad$ Remote sensing of local density gradients over distances comparable with particle gyroradii. Species dependent structures in gradients can be studied, gradient motions can be resolved to half the spinperiod $(\delta t=1.5 \mathrm{sec})$.

- Determination of major ion species (H, He, CNO) in the energetic plasma component (LD). A special operational mode of LD allows identification and analysis of energetic neutral atoms (ENA).

- Characterization of magnetic field line topologies using fast motion of energetic electrons (in particular, BD).

The LD/BD instrumentation consists of two independent detector systems with different detection techniques. The LD spectrometer employs time-of-flight/energy analysis to determine the particle mass and uses the concept of a rotating "projection camera" to obtain contiguous three-dimensional intensity distributions. The BD spectrometer is based on the $\Delta E \times E$ method. Three identical telescopes with solid state detectors cover a significant fraction in velocity space. The digitized outputs from all instruments (LD, BD, MI, and $\mathrm{HI}$ ) are received by a common digital processing unit (HEP-E) for evaluation and formatting. An overall block diagram of the HEP-E complement with emphasis on the LD/BD dual spectrometer is shown in Fig. 1.

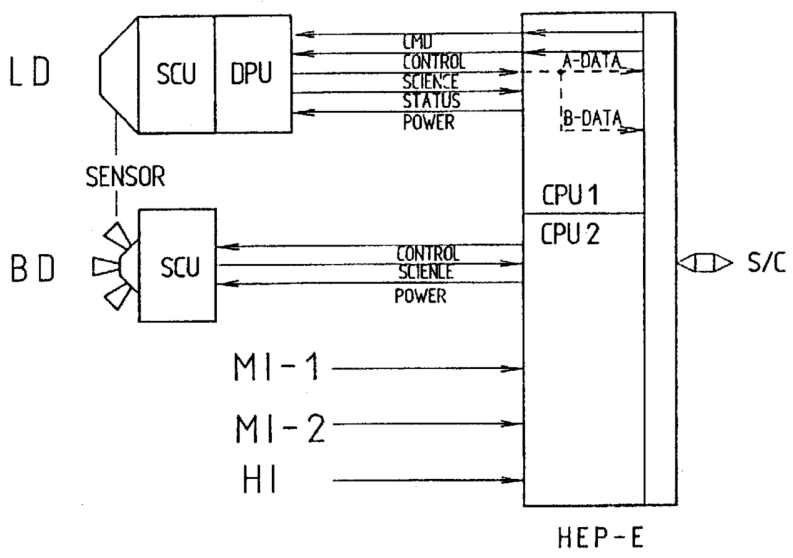

Fig. 1. An overall block diagram of the HEP complement with emphasis on the LD/BD dual spectrometers. 


\subsection{The LD spectrometer (Low energy particle Detector)}

The LD instrument is physically a single structure which contains; the sensor system, the front-end electronics (called SCU), and the digital processing unit (DPU) with the interface to the HEP-E unit in the back of the box. The LD sensor system is composed of three identical SCENIC heads S1, S2 and S3. Overall dimensions of the box and the positions of the three systems in the instrument reference system are shown in Fig. 2.

The center piece of the LD sensor system is the so-called SCENIC detector head. The acronym stands for "spectroscopic camera for electrons, neutral and ion composition". In essence SCENIC is a miniature telescope composed of a time-of-flight (TOF) and energy $(E)$ detection system. The noval aspect is the linear imaging of flux distributions and the capability to identify energetic neutral atoms (ENA) in a certain energy band.

The particle identifying function of the SCENIC spectrometer is obtained from a two-parameter measurement: The particle velocity $(V)$ and the energy $(E)$ are measured as independent quantities, the particle mass $A$ is then uniquely determined from the equation $A \sim E / V^{2}$. Actually, the velocity detector measures the flight-time $(T)$ the particle needs to travel a known distance in the detector geometry.

Figure 3 shows somewhat simplified cross-sections of the SCENIC detector telescope drawn to scale. A particular feature is the triangular structure with a $60^{\circ}$ opening angle. The energy measuring solid state detectors (SSD) are mounted in the apex at the rear of the system. A group of two SSDs (the energy detector and the back detector) is combined in an anticoincidence condition for high energy electron detection. The flight-time $(T)$ measuring system is the entry element of the telescope. It is essentially composed of a thin foil $\left(5 \mu \mathrm{g} / \mathrm{cm}^{2}\right.$ carbon foil with $\mathrm{Al}$ coating) and the front surface of the energy detector. The distance between the foil and the energy detector along the line of symmetry is the nominal flight path $(s=34 \mathrm{~mm})$ for the $T$-measurement. Particles passing through the telescope release "secondary electrons" from the entry foil. The secondary electrons are accelerated and directed to a microchannel plate (MCP) for detection. The MCP output signal constitutes the START signal for the $T$-measurement. Details of the isochronous secondary electrons transfer to the START-MCP are shown in the upper cross section of Fig. 3. Upon impact of the particle on the energy detector secondary electrons are ejected from the surface as well. These secondary electrons are trasnsferred to the STOP-MCP by a technique similar to the start electrons. The STOP signal completes the $T$ measurement.

The energy $E$ of the incident particle, reduced by the loss in the START foil, is measured in the energy detector. For sufficient high energies, the particle is able to penetrate the energy detector and to strike the back detector. The back detector signal leads to the elimination of the event from analysis.

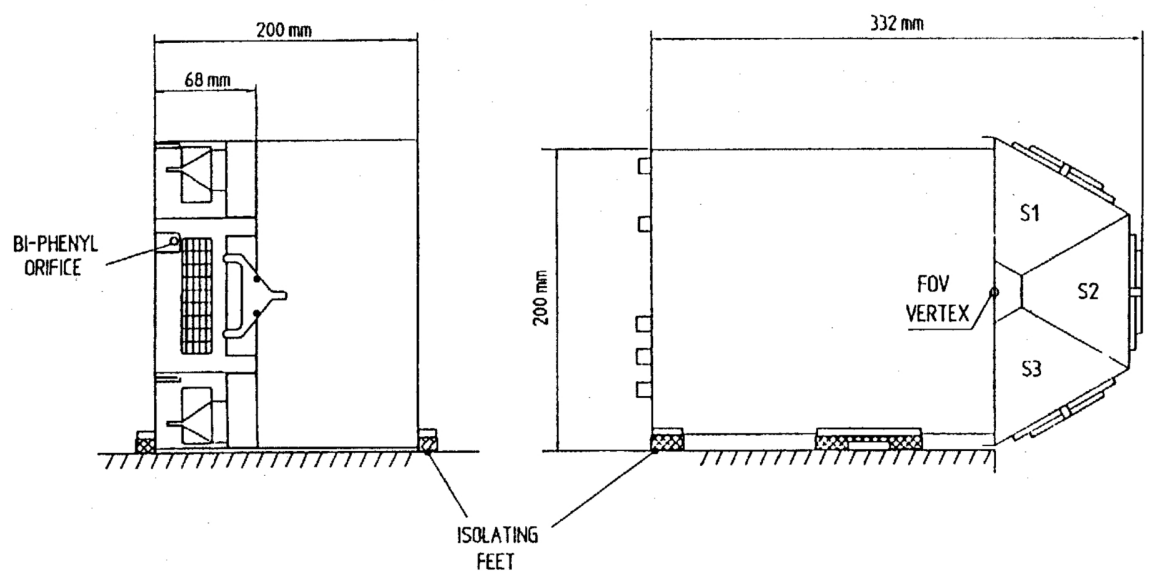

Fig. 2. Outer envelopes of the LD spectrometer with some principal dimensions. 


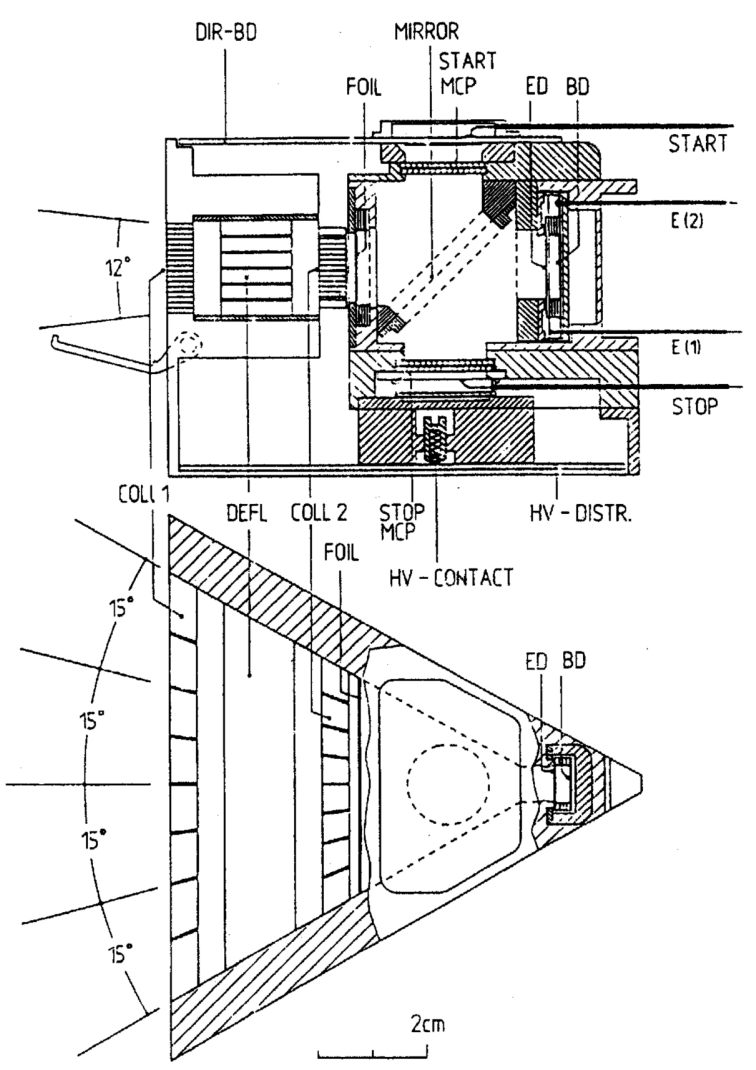

Fig. 3. Simplified cross sections of the SCENIC detector telescope drawn to scale (ED: Energy Detector, BD: Back Detector, COLL: Collimator, DEFL: Deflector).

The design of the SCENIC head is such that the secondary electrons transfer to the START-MCP is not only isochronous but also position preserving: Four read-out anodes (not shown in Fig. 3) correspond to four contiguous segments on the entry foil and each of these forms a $12^{\circ}$ by $15^{\circ}$ viewing cone with the energy detector in the back of the system. In a sense this geometry can be considered a degenerated case of a "projection camera" with only one pixel in the back plane. Incident particles are strongly collimated before they reach the $T / E$-telescope. Two microchannel collimators define a highly anisotropic field-ofview with $12^{\circ}$ lateral and $60^{\circ}$ polar opening. A set of plates between the collimating elements with alternating potentials $(0$ and $+\mathrm{Udef})$ forms a linear electrostatic deflector. The primary purpose of DEFL is to protect the instrument from overloads due to large fluxes of low energy particles (e.g. solar wind plasma). However, the relative high gain of the active collimator (approx. $g=10$ ) allows efficient separation of energetic neutral atoms from ions for energies up to $100 \mathrm{keV}$. This energy band is generally considered important for magnetospheric neutrals produced in the ring current region.

The fraction in $E-T$ space for which identification of nuclides is possible extends in energy $(E)$ from $30 \mathrm{keV}$ (lower threshold) up to $1500 \mathrm{keV}$ (upper end of the linear range in the energy channels) and in timeof-flight $(T)$ from $0 \mathrm{nsec}$ to $80 \mathrm{nsec}$. Particle events $(E, T)$ falling into this parameter field are fully identified. In addition, unique species information can be extracted from a $T$-measurement alone for energies between $1500 \mathrm{keV}$ and $4000 \mathrm{keV}$ if certain logic conditions are met. Incident particles with energies above $30 \mathrm{keV}$ and flight-times $T \approx 1 \mathrm{nsec}$ are identified as electrons. The probability $P(E)$ for full absorption in a $300 \mu \mathrm{m}$ energy detector (Berger et al., 1969), suggests a useful energy range from 30 to $400 \mathrm{keV}$ for 
electron detection. Electrons penetrating the energy detector create a veto signal in the back detector which results in the elimination of these events from subsequent analysis (the same applies for high energy nuclei).

As already mentioned earlier and as shown in Fig. 4 the LD sensor system is composed of three identical SCENIC heads in a configuration such that contiguous coverage over $180^{\circ}$ in the polar angle is achieved if this angle is defined with respect to the GEOTAIL spin axis. The sectored rotation of the spacecraft provides the completing azimuthal coordinate. Selected LD parameters are summarized in Table 1.

\subsubsection{The digital processing unit (DPU)}

The output signals of the three SCENIC heads in the LD spectrometer are fed to a segment of the electronic system which provides proper analog amplification, signal shaping, event definition logic,

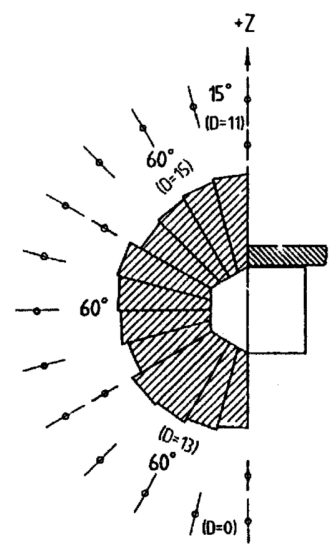

LD

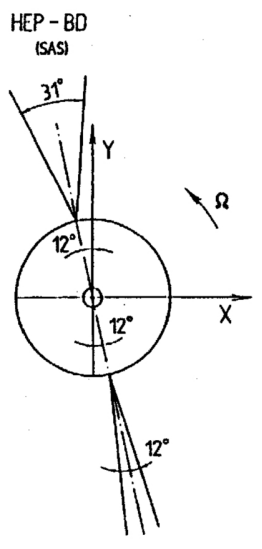

HEP - LD



$B D$

Fig. 4. The diametric positions of the LD and BD instruments in the spacecraft and the field-of-view in the polar and azimuthal directions.

Table 1. Characteristic parameters of the LD sensor system.

\begin{tabular}{ll}
\hline Energy range (keV) & $30-1500(4000)$ \\
Hydrogen & $80-4000$ \\
Helium & $160-1500$ (Oxygen) \\
CNO & $30-400$ \\
Electrons & $10-100$ \\
ENA* & $1,4,12-16,28-56$ \\
Mass classes (amu) & $4($ Oxygen) \\
Mass resolution (A/dA) & $+/-6^{\circ} \times 180^{\circ}$ \\
Field-of-view & $180^{\circ} / 12$ \\
Angular coverage & $360^{\circ} / 16$ \\
$\quad$ Polar (Range/Intervals) & $0-10 / 16$ \\
$\quad$ Azimuthal (Range/Sectors) & \\
Deflection voltage (kV) & $2.4 \times 10^{-1} / 2 \times 10^{-2}$ \\
$\quad$ Range/Steps & \\
Geometric factor $\left(\mathrm{cm}^{2} . \mathrm{sr}\right)$ & \\
Total/Differential &
\end{tabular}

*ENA: Energy Neutral Atoms. 
Table 2. Definition of the Science Data and prime scientific objective.

\begin{tabular}{|c|c|c|c|c|c|}
\hline Data type & Particle species & No. of E-Ch. & $\begin{array}{c}\text { Angular intervals } \\
\text { (Polar) }\end{array}$ & $\begin{array}{l}\text { Azimuthal } \\
\text { sector }\end{array}$ & Scientific objective \\
\hline I-SPCT & $\mathrm{H}, \mathrm{He}, \mathrm{CNO}$ & $8 \mathrm{~d}$ & $\mathrm{i}$ & $\mathrm{i}$ & Energy-spectrum \\
\hline I-FB & $\mathrm{H}$ & $2 w$ & $3 \times 60^{\circ}$ & 16 & 3-d distribution, fast \\
\hline I-3Ds & $\mathrm{H}, \mathrm{He}, \mathrm{CNO}, \mathrm{Si}$ & $8 d$ & $12^{\circ} \times 15^{\circ}$ & 16 & 3-d distribution (1) \\
\hline I-3DF & $\mathrm{H}, \mathrm{He}, \mathrm{CNO}$ & $2 w$ & $3 \times 60^{\circ}$ & 16 & 3-d distribution, fast \\
\hline I-MTRX & all & all & $\mathrm{i}$ & $\mathrm{i}$ & A - E/A distribution \\
\hline E-FB & Electrons & $2 w$ & $3 \times 60^{\circ}$ & 16 & 3-d distribution, fast \\
\hline E-3DS & Electrons & $8 \mathrm{~d}$ & $3 \times 60^{\circ}$ & 16 & 3-d distribution \\
\hline E-3DS+ & Electrons & $8 d$ & $12^{\circ} \times 15^{\circ}$ & 16 & 3-d distribution (1) \\
\hline $\mathrm{DE}$ & All & 256 & $12^{\circ} \times 15^{\circ}$ & 16 & High resolution particle data \\
\hline
\end{tabular}

$\mathrm{d}=$ narrow differential energy channels, $w=$ wide energy channels, $\mathrm{i}=$ integral, $\mathrm{A}=$ atomic mass $(\mathrm{amu}), 3-\mathrm{d}=\mathrm{three}$ dimensional.

(1)High resolution in energy and angle.

Note: E-3DS or E-3DS+ alternatively.

control functions for configuring the detector system, and the interface to the internal digital processing unit. An incident particle generates a set of signals (analog and digital) in the energy $(E)-$, the time $(T)$ and the direction (DIR) channel.

The digital processing unit (DPU) controls the signal conditioning unit (SCU) and the sensor system, and evaluates the primary event information $(E, T, \mathrm{DIR})$ to compress the obtained data to a level which is comparable with the telemetry capacity, and arranges the output data (Science Data) in the format of an experiment data block (EDB). The science data are grouped in ion data (I-SPECT, I-FB, I-3DS, I-3DF, MTRX) and electron data (E-FB, E-3DS, E-3DS+). These data types are obtained from the on-board classification process. In addition, and independent of the classification algorithm a number of so called direct events is transmitted with full resolution in $(E, T, \mathrm{DIR})$. Definitions of the various science data and brief descriptions of the scientific intention are given in Table 2.

\subsection{The BD spectrometer (Burst Detector)}

The HEP-BD instruments are designed to observe the high energy electrons, protons and heliums. The objectives are: 1) particle acceleration mechanism in the geotail region, 2) propagation effects, 3) reconnection process of the geomagnetic line of force to accelerate the particle in the near-earth orbit, and 4) energy spectrum and pitch angle distributions of high energy particles. The HEP-BD instruments have a large geometrical factor to obtain high time/energy/angular resolutions of low intensity particles in the geotail region. The $\mathrm{BD}$ spectrometer consists of three silicon $\Delta E \times E$ silicon detector telescopes and measures electrons in the energy range $0.12-2.5 \mathrm{MeV}$, protons and helium $0.4-3.5 \mathrm{MeV}$, protons $3.5-35 \mathrm{MeV}$, and helium 3.5-35 MeV/u. The instruments have rectangular opening angles $30^{\circ} \times 45^{\circ}$ and a geometrical factor $0.76 \mathrm{~cm}^{2} \mathrm{sr}$ for each telescope. This geometrical factor is 36 times larger than the geometrical factor $\left(0.02 \mathrm{~cm}^{2} \mathrm{sr}\right)$ of each LD sensor. These wide coverage of energy and large geometrical factor make possible to observe the large intensity variations in the geotail region.

\subsubsection{Characteristic of $B D$ sensor}

Identification of electrons, protons and helium ions by $\mathrm{BD}$ instrument is based on the $\Delta E \times E$ silicon telescope system. The amount of energy deposited in the passing detector is denoted by $\Delta E$ and the amount of energy deposited in the stopping detector is denoted by $E$. The total energy is then $E_{0}=\Delta E+E$.

The energy loss $\Delta E$ of a charged particle of energy $E_{0}$, mass $M$ and charge $z$ in thin passing detector is; 
(a)

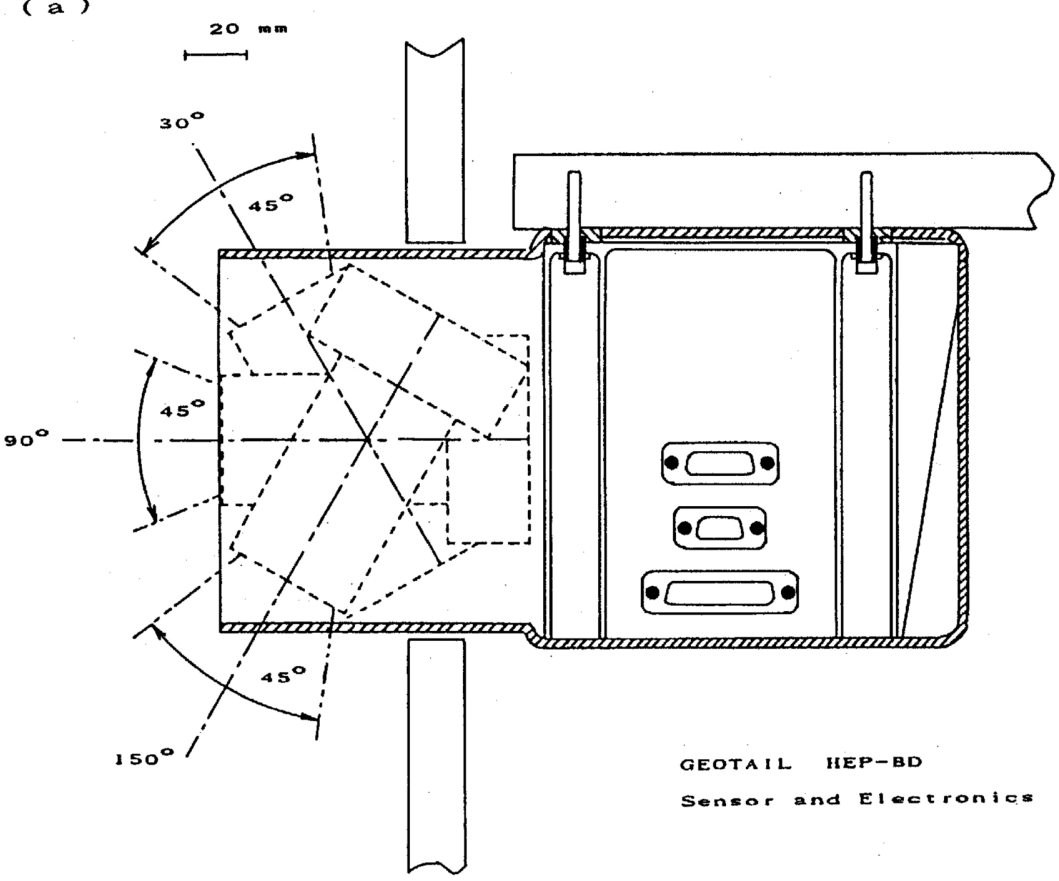

(b)
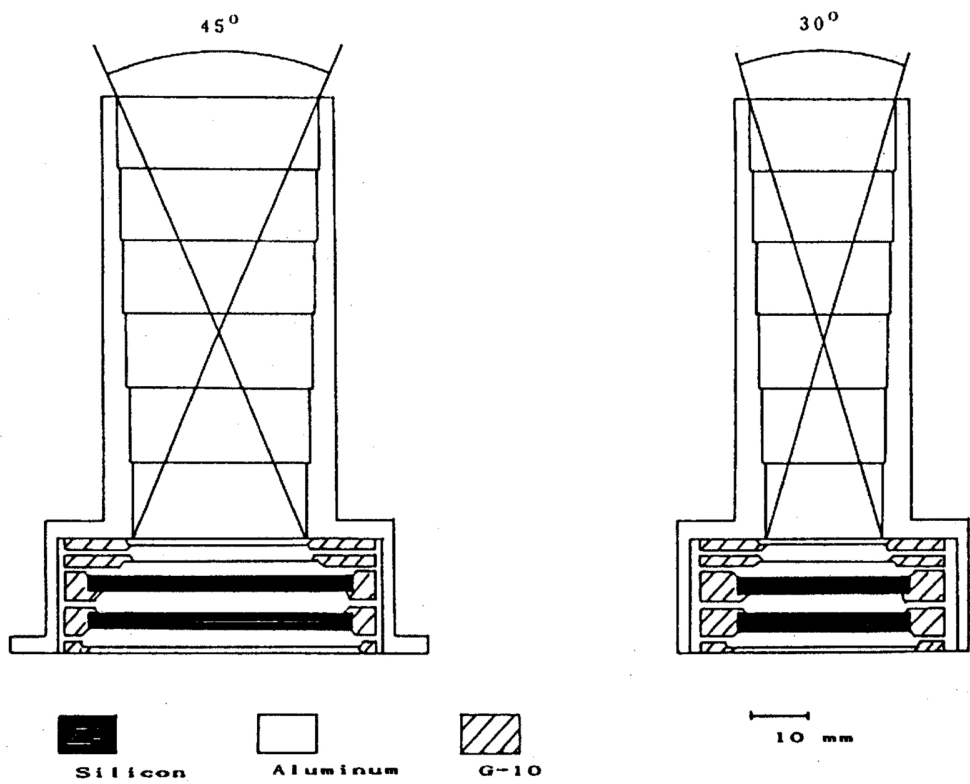

HEP-BD Sensors

Fig. 5. (a) Three telescopes are installed to the GEOTAIL satellite looking for $30^{\circ}, 90^{\circ}$ and $150^{\circ}$ to spin axis. (b) Cross sectional view on one of three BD telescopes. Each telescope consists of five silicon detectors; a $\Delta E$ detector, three $E$ detectors, and an anticoincidence detector. 
Table 3. Characteristics of GEOTAIL HEP BD spectrometer. BD spectrometer consists of three telescopes. Each telescope has rectangular opening window.

\begin{tabular}{lll}
\hline Particle & Electron & $0.20-2.5 \mathrm{MeV}$ \\
& Proton + Helium & $0.4-3.5 \mathrm{MeV} / \mathrm{u}$ \\
& Proton & $3.5-35 \mathrm{MeV}$ \\
& Helium & $3.5-35 \mathrm{MeV} / \mathrm{u}$ \\
Telescope & 3 units & \\
Look direction & $30^{\circ}, 90^{\circ}, 150^{\circ}$ to spin axis & \\
Opening angle & $30^{\circ} \times 45^{\circ}$ & \\
Geometrical factor & $0.76 \mathrm{~cm}^{2} \cdot \mathrm{sr}$ each telescope \\
Telemetry bit rates & $3072 \mathrm{bps}$ Real Mode \\
& $640 \mathrm{bps}$ Record Mode \\
\hline
\end{tabular}

$$
\Delta E \propto M z^{2} / E_{0}
$$

This relationship is used to identify electrons, protons and helium ions. The telescope consists of a thin $\Delta E$ passing detector, and three stopping $E$ detectors and an anticoincidence detector. The $\mathrm{BD}$ instrument consists of three $\Delta E \times E$ telescopes (BD-I, -II, and -III). The look directions are $30^{\circ}, 90^{\circ}$ and $150^{\circ}$ to spin axis as shown in Fig. 5(a). The characteristics of BD sensor is summarized in Table 3.

The cross-sectional view of the telescope is shown in Fig. 5(b). An $80 \mu \mathrm{m}$ ion implantation silicon detector with sensitive area $20 \times 30 \mathrm{~mm}^{2}$ is used for the $\Delta E$ passing detector, the stopping $E$ detector are a $0.4 \mathrm{~mm}$ ion implantation silicon detector with sensitive area $22 \times 32 \mathrm{~mm}^{2}$ and two $3 \mathrm{~mm} \mathrm{Si}$ (Li) detectors with sensitive area $27 \times 43 \mathrm{~mm}^{2}$, and $0.4 \mathrm{~mm}$ silicon surface barrier detector with sensitive area $27 \times 43$ $\mathrm{mm}^{2}$ which is used as an anticoincidence detector.

\subsubsection{Data processing unit}

BD electronics system consists of analog signal processing unit, pulse height analyzing unit and digital signal processing unit. Charge sensitive pre-amplifiers and high voltage supplies for SSD of BD, $\mathrm{MI}$ and $\mathrm{HI}$ are contained in each sensor unit. Other parts of electronic system are contained in HEP-E. The analog signal processing part and ADC part of pulse height analyzing unit stand alone for each sensor. Histograms of observed data are made as a function of the energy of each particle, the angular distribution and the spin of satellite. Observations of the on-board HEP-BD instruments have three operational modes: Mode-A (Real and Contingency), Mode-B (Record Mode) and Mode-C (Record Mode). Mode-A consists of high time resolution mode. The energy spectrum of electrons, protons and helium ions are obtained in 16 sector steps for every $3 \mathrm{sec}$ spin period. The energy channel steps are 6 steps for electrons, 8 steps for protons, and 4 steps for helium ions in Mode-A. In the Mode-B, the energy spectra of electrons, protons and helium ions are obtained in 8 sector steps for every $6 \sec (2 \mathrm{spins})$, and in the Mode-C, in 16 sector steps for every $12 \mathrm{sec}$ ( 4 spins). The energy steps in Mode-B and -C are 5 steps for electrons, 6 steps for protons and 4 steps for helium ions. The difference between Mode-B and Mode-C is the sampling time $6 \mathrm{sec}$ or $12 \mathrm{sec}$. Summary of data acquisition mode is shown in Table 4. Every BD data group is processed in the spin synchronized format frame. The format is started by the sun pulse. The azimuthal angle is divided into 16 sectors per spin in Mode-A, 8 sectors per 2 spins in Mode-B, and 16 sectors per 4 spins in Mode-C.

\subsection{The LD/BD complement and initial results}

The previous sections provided a comprehensive account of technical concepts and major functions of the two particle spectrometer HEP-LD and HEP-BD. The rather different detection techniques employed in the instruments emphasis by their very nature different energy ranges in magnetospheric plasmas. Figure 6 is a graphic representation of the coverage in mass $(A)$ versus energy/nucleon space for ions and electron. The LD spectrometer is designed to identify dominant magnetospheric elements or 
Table 4. Data analysis mode of HEP BD. BD has 3 modes; Time high resolution mode (Mode A), 8 sectors mode (Mode B), and 16 sector mode (Mode C).

\begin{tabular}{|c|c|c|c|}
\hline Format & Words and frames & Date & ysis mode \\
\hline $\begin{array}{l}\text { Format } 2 \text { and } 3 \\
\text { (Real and } \\
\text { Contingency mode) }\end{array}$ & $\begin{array}{l}6 \text { Word/Frame } \\
64 \text { Frame/Spin }\end{array}$ & $\begin{array}{l}\text { Mode } A^{*} \\
\text { (Time high } \\
\text { resolution mode) }\end{array}$ & $\begin{array}{l}1 \text { Spins, } \\
3 \mathrm{sec} \text {, } \\
16 \text { Sectors }\left(=360^{\circ} / 16\right)\end{array}$ \\
\hline \multirow[t]{2}{*}{$\begin{array}{l}\text { Format } 1 \\
\text { (Record mode) }\end{array}$} & $\begin{array}{l}5 \text { Words/Frame } \\
16 \text { Frame/Spin }\end{array}$ & Mode B* & $\begin{array}{l}2 \text { Spins, } \\
6 \text { sec, } \\
8 \text { Sectors }\left(=360^{\circ} / 8\right)\end{array}$ \\
\hline & & Mode C* & $\begin{array}{l}4 \text { Spins, } \\
12 \mathrm{sec} \text {, } \\
16 \text { Sectors }\left(=360^{\circ} / 16\right)\end{array}$ \\
\hline
\end{tabular}

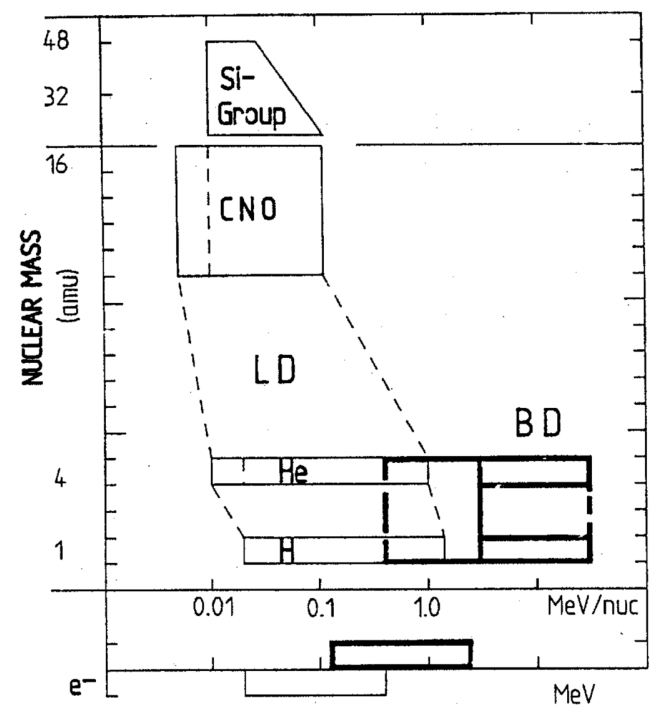

Fig. 6. Graphic representation of the coverage in mass versus energy/nucl space for ions and electron.

groups of elements with a good resolution was compromised deliberately. The time-of-flight technique with SE emitting foils opens the possibility to achieve lower energy limits than is possible with solid state detectors. As seen in Fig. 6, the $\Delta E \times E$ technique used in BD provides a very important extension to high energies with a good overlap to the LD range for medium energies.

The diametric positions of the two instruments in the spacecraft and the field-of-view in the polar and azimuthal directions are indicated in Fig. 4 (it is worth noting that the SAS sensor which provides the azimuthal reference pulses for $\mathrm{LD} / \mathrm{BD}$ is collateral with $\mathrm{BD}$ ). This provides an ideal geometry for measuring fast flux variations due to the motion of density gradients or due to the development of flow anisotropies. Inspection of Fig. 5(a) suggests that measurements with time resolution of $1.5 \mathrm{sec}$ should be possible for selected energy band and particle species.

\subsubsection{Initial results of $L D / B D$ spectrometers}

HEP-BD and -LD instruments were successfully turned on, on August 17 and September 22, 1992, 
respectively. Both instruments have been operating well, though some anomalies have been found in the acquired data, as summarized below.

(1) For the BD sensor system, the obtained shape of energy spectrum is not fully consistent among three telescopes looking at different directions. This is found to be true for the case of very isotropic solarflare particle events. Calibration studies are presently under way. This anomaly does not affect the observed azimuthal distribution of particles.

(2) The probability of electrons triggering the time-of-flight circuit of the LD sensor system seems to be very low. The efficiency for ions is much higher, but full calibration for the three detectors (particularly, for the one looking at the equator) is not completed yet. (We may have to equalize the efficiency by adjusting high voltages applied to the MCP.) The energy and counting-rate information from SSDs is very consistent among the three sensor systems.

Since the evaluation and adjustment of these anomalies have not been completed yet, we will limit ourselves in this report to the temporal variations in the energy-integrated counting rate and the azimuthal anisotropy of particles. This limited information alone has revealed interesting aspects of particles accelerated in the earth's magnetotail at the time of substorm onset.

In passing, in order to demonstrate how the time-of-flight technique is functioning for $L D$, we show in Fig. 7(a) typical scatter plots of ions in the energy $(E)$ versus time-of-flight $(T)$ diagrams. These diagrams are drawn based on the DE data in the telemetry described earlier in Table 2. Combination of $E$ and $T$ for a reduced number of ions is selected on-board as DE (Direct Events) for direct data transmission; two hour accumulation of such data is plotted here. Date and time of observation is indicated in the figure. Different panels correspond to different azimuthal sectors. The three slant lines in each panel indicate the

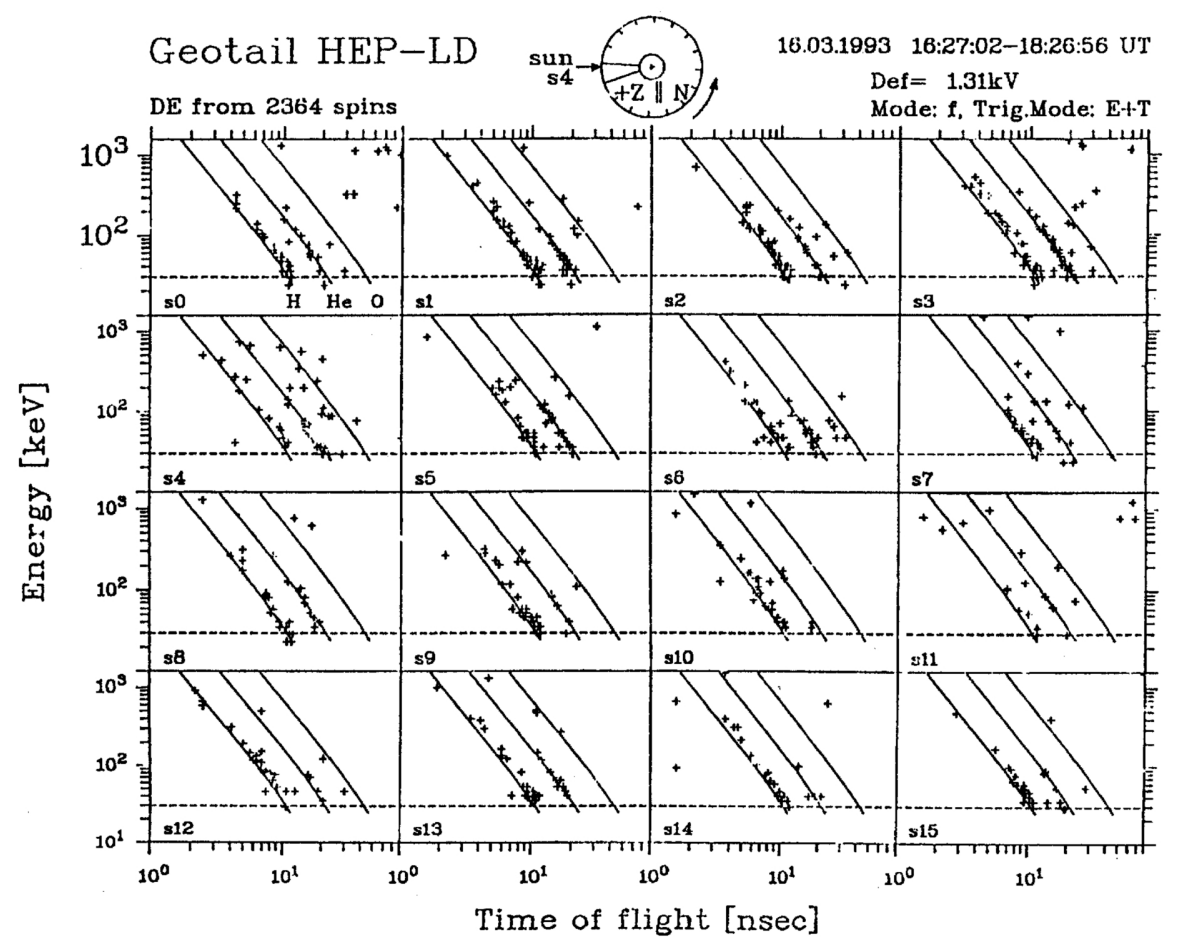

Fig. 7(a). Energy $(E)$ versus Time of Flight $(T)$ scatter plots obtained from LD measurements. Plotted ion events are those which were selected on board for the direct transmission and subsequent ground analyses. Three slant lines in each diagram correspond to the expected relationship for protons, Heliums, and CNO group ions, respectively. Different panels correspond to different azimuthal sectors. Data were accumulated for about two hours (16:27:02-18:26:56 UT, March 16, 1993). 
expected $E-T$ relationship for protons, helium, and CNO group, respectively. These diagrams demonstrate that the separation of observed $E$ and $T$ signals among each particle subgroup is sufficient for the particle identification. The identification is done automatically by the on-board microprocessor, though additional information in such forms as DE and SGL is included in the telemetry for evaluation on the ground.

The substorm we will be discussing occurred on January 31, 1993, when GEOTAIL was near the plasma sheet boundary about $50 \mathrm{Re}$ down the tail. Figure 7(b) shows the energy-integrated counting rate of $\mathrm{BD}$ detectors for the $8 \mathrm{hr}$ of real-time operation on this day. Ground magnetograms show that this day was a moderately active day and that there was continuous magnetic activity in the auroral zone. For this $8 \mathrm{hr}$ interval, five substorm expansion onsets have been determined from the particle injection time monitored at the geostationary orbit by the Japanese GMS-4 satellite (courtesy Dr. T. Nagai). We will discuss the earliest onset that occurred at 14:10:36 UT as determined from the GMS-4 data. GEOTAIL was in the southern part of the tail at $(-50.0,-7.5,-4.3)$ Re in the GSM coordinates around this time. We note in Fig. 7(b) that two major peaks are presented in the BD counting rate around the time of this substorm onset. The first one preceded the onset and therefore was not caused by the substorm onset itself (though it may be related to the plasma processes operating in the growth phase). As a matter of fact, we will see below that only the second one which occurred after the substorm onset was accompanied by a strong anisotropy of streaming particles.

Expanded views of BD counting rates and anisotropy for the 30 minute interval from 1400 to 1430 UT are shown in Fig. 7(c) together with the total magnitude and $z$ component records of the magnetic field. In the top and third panels total counting rates of electrons $>150 \mathrm{keV}$ and protons $>250 \mathrm{keV}$ are plotted respectively, and the color-coded diagram immediately below each counting-rate plot shows the azimuthal anisotropy of each species. The anisotropy is represented by the color-coded flux for 16 azimuthal sectors arranged vertically; the vertical scale is such that the top and bottom of the panel correspond to the spin phase of the detector looking toward the sun, and the center does the spin phase

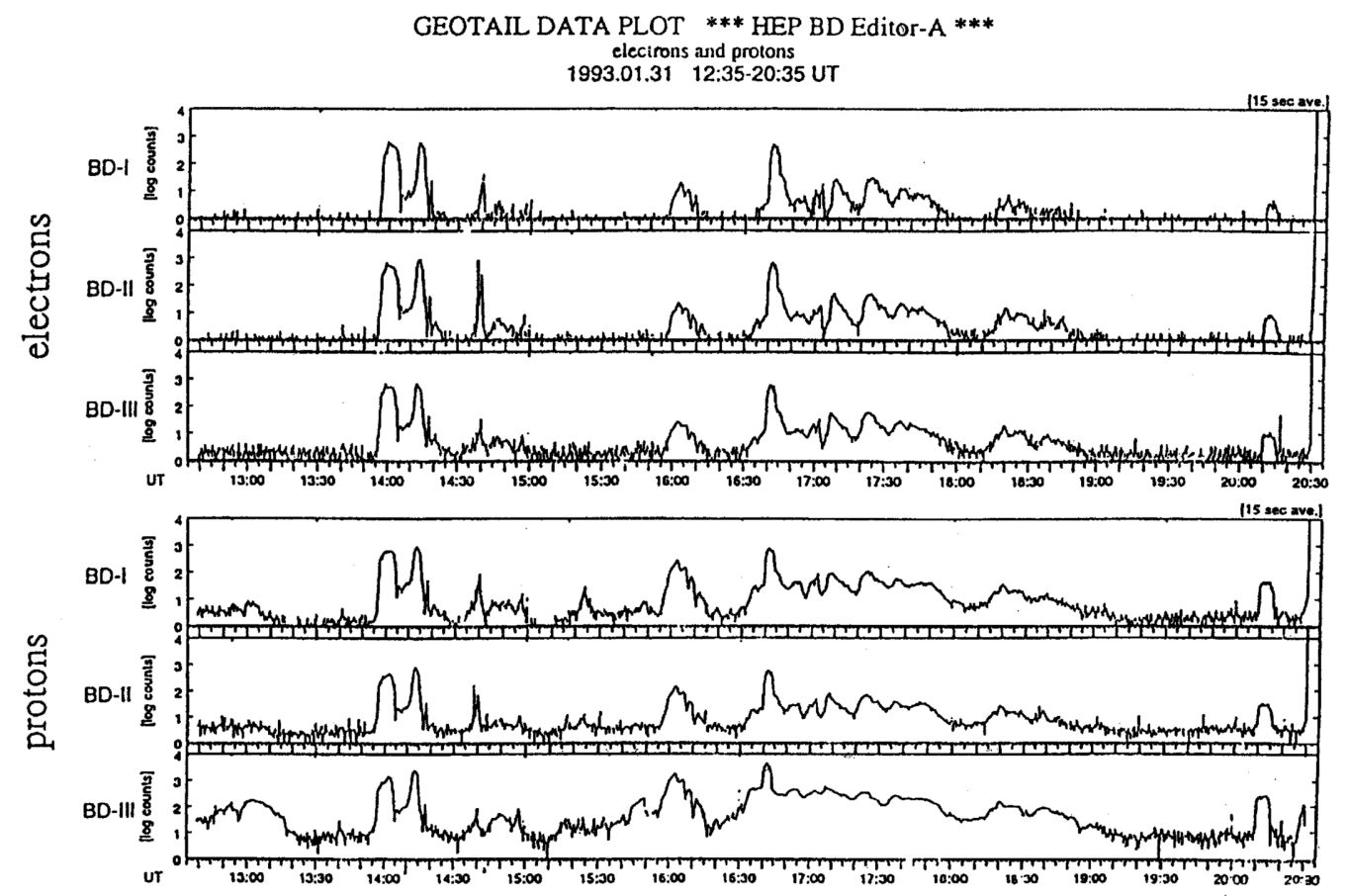

Fig. 7(b). Integrated flux of protons ( $>250 \mathrm{keV})$ and electrons $(>150 \mathrm{keV})$ from three BD sensors. About eight hours of real-time transmission are shown for the day January $31,1993$. 
looking towards the tail. The color shade goes from dark blue to red as the particle flux increases, with the maximum flux of about $10^{3} \mathrm{counts} / \mathrm{sec}$ represented by red. The magnitude (the upper curve) and the $z$ component (the lower curve) of the magnetic field are given in the bottom panel.

It is immediately apparent from the comparison of the BD counting rate and magnetic field data that the two major peaks noted earlier in Fig. 7(b) (the start of the first one was prior to 14:00 UT) were each accompanied by a depression of the magnetic field, indicating that they were due to a partial entry of the satellite into the plasma sheet. In anisotropy panels, the first entry was characterized by a more or less isotropic distribution of electrons and protons, while the second entry was characterized by an intense bidirectional anisotropy directed roughly along the sun-earth line. It is notewarthy that the second entry to the plasma sheet occurred at about 14:11:30 UT, less than one minute after the substorm onset recorded at the geostationary orbit. The magnetic field record shows a bi-polar signature in the $z$ component; the magnetic field was directed northward prior to and during the period when the satellite was in the plasma sheet, and then tipped southward as the satellite left the plasma sheet and entered the lobe. We interpret this bipolar signature in $B z$ as a typical signature of the plasmoid passage. (The fact that the satellite left the plasma sheet when the field turned southward may indicate that the earthward portion of the plasmoid (where $B z$ is southward) is thinner than the forward portion (where $B z$ is northward), but the detailed discussion of the shape of the plasmoid is beyond the scope of this paper.) We also note that the satellite probably grazed the outer portion of the plasmoid since the magnetic field magnitude never approached zero.

For the sake of comparison, the energy-integrated counting rate of $>30 \mathrm{keV}$ particles (protons plus electrons) derived from LD is plotted in the upper panel of Fig. 8(a). The counting rate of north, equator, and south-looking detectors are distinguished by different line colors, i.e., blue, red, and green, respectively. The azimuthal anisotropy of particles from the equator-looking detector is shown in the



Fig. 7(c). Integrated flux of electrons (top panel) and protons (third panel) are shown for the period 14:00-14:30 UT, January 31, 1993. Color-coded diagrams show azimuthal anisotropy of particles as explained in the text. The bottom panel shows the magnitude and the $z$ (north-south) component of the observed magnetic field. 
lower part of the figure for selected time intervals. The upper panel shows that, around the onset time of 14:10:36 UT, the particle flux recorded by all three detectors began to increase gradually as the satellite entered the plasmoid. A precursory disturbance is seen just before the onset, and is accompanied by an enhanced flux from the north. The flux finally peaked at around 14:14 UT; it is noteworthy that the counting rate of the equator-looking detector exceeded those of other detectors by a factor of two for a short time interval around the time of the peak flux. Before the substorm onset, the azimuthal anisotropy of particles was almost isotropic (interval A). As the particle flux increased after the onset, tailward streaming anisotropy developed (i.e. strong flux is observed when the detector looks forward the earth, which is to the left). The anisotropy became particularly strong during the two minutes (14:13:0014:15:00) centered at the time of peak total flux (intervals D-F). In this time period, the particles were collimated so well, that only one azimuthal sector $\left(45^{\circ}\right.$ wide) responded to this increase. When the satellite was exiting the plasma sheet (interval $\mathrm{H}$ ), it observed the particle flux perpendicular to that observed earlier; we attribute this to the density gradient effect at the plasma sheet boundary.

Similar snapshots of azimuthal anisotropy obtained from BD are shown in Fig. 8(b). Upper two rows of diagrams are for electrons $(>150 \mathrm{keV})$ and lower two rows for protons $(>250 \mathrm{keV})$. The format of presentation is the same as in Fig. 8(a) except that there are 16 azimuthal sectors in the equatorial plane. It is seen that around the time of the maximum flux (panels (c) to (f)), the particles (both electrons and protons) were bi-streaming in the $\mathrm{BD}$ energy ranges, as opposed to the single beaming of lower energy particles detected by LD. Toward the end of the period (panel (g)), the particles flow direction detected by $\mathrm{BD}$ is even opposite to that for LD. The bi-stream distribution started about $30 \mathrm{sec}$ earlier than the strong tailward collimation of LD particles. This bi-stream anisotropy is almost aligned with the magnetic field direction observed simultaneously.

It is interesting that the single-stream anisotropy of LD-energy-range particles coexisted with the bistream anisotropy of BD-energy-range particles, sharing the same field line with them. The bi-stream type anisotropy is usually taken as evidence that the field line by which the particles are guided is closed. This idea is consistent with the interpretation that the entry of GEOTAIL to the plasma sheet is caused by an encounter with a plasmoid constituting looped field lines. Of course, in order to be actually observed, it is needed that there had been enough time for the particles initially ejected tailward to get to the tailward end of the loop, be turned around, and come back to the position of the satellite to be superposed on the particles newly arriving from the earth direction. This last condition is probably satisfied because ions (assumed to be protons) of the lowest energy (250 keV) detected by BD can travel as long as 120 Re within two minutes that had past between the substorm onset and the time of observation. (We assume that BDenergy-range particles are not significantly scattered along their path.) Then the question to ask is why the lower energy particles observed by LD did not show such a bi-stream anisotropy. One of the possibilities is that the lowest energy ions at $30 \mathrm{keV}$ had insufficient time to make a round trip along the loop while the satellite was in the closed field line region. For this to happen for $30 \mathrm{keV}$ protons, the length of the loop in the tailward direction should be longer than $80 \mathrm{Re}$ if we assume the duration of the satellite's stay in the closed field line region to be four minutes. This possibility cannot be denied entirely, but we prefer another possibility that as opposed to $\mathrm{BD}$-energy range particles, $30 \mathrm{keV}$ protons are significantly scattered to be isotropic in the frame of flowing plasma. As a matter of fact, it has been reported in a number of literatures (e.g. Scholer et al., 1984; Daly et al., 1984) that anisotropy of energetic particles in the tail is consistent with the interpretation that particles around $30 \mathrm{keV}$ represent the high-energy tail of the shifted-Maxwellian distribution or shifted-power-law distribution moving with the bulk speed of the plasma. If this interpretation is correct, our observation suggests that the inner closed-field-region of plasmoid as grazed by GEOTAIL was moving faster than the surrounding region.

In conclusion, in one case examined in this paper, the plasmoid observed at $50 \mathrm{Re}$ down the tail contained high energy particles that were roughly collimated along the sun-earth direction. The detailed anisotropy of the particle distribution is found to differ for different energies. We have tentatively interpreted the single stream observed at low energies to reflect the bulk motion of the plasmoid, and the bi-stream anisotropy at higher energies to reflect a higher-energy population which was accelerated at the 




anisotropy

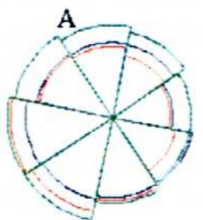

E

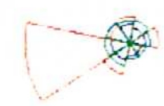



F



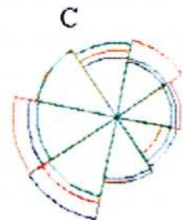

G

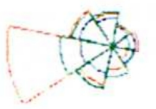

D

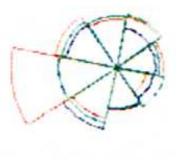

$\mathrm{H}$

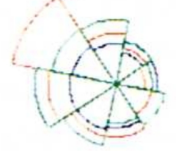

Fig. 8(a). Integrated particle flux (ions plus electrons) for the $>30 \mathrm{keV}$ energy range is plotted for the period 14:00-14:30 UT, January 31, 1993. LD-1 (North), 2 (Equator), 3 (South) detectors are distinguished by blue, red and green lines, respectively. Azimuthal anisotropies shown in the lower panel are for the following time intervals: (a) 14:11:00-14:15:00 UT, (b) 14:12:00-14:12:30 UT, (c) 14:12:30-14:13:00 UT, (d) 14:13:00-14:13:30 UT, (e) 14:13:30-14:14:00 UT, (f) 14:14:00$14: 14: 30$ UT, (g) 14:14:30-14:15:00 UT, (h) 14:15:00-14:15:30 UT. The plane of the figure is the spin plane, which is almost equivalent to the solar-ecliptic plane. The sun is to the left.

source in the filed direction and then streamed back and forth relative to the plasma along the closed field lines of the plasmoid.

\section{The Energetic Isotope Telescopes (MI and HI)}

The direct observations of solar energetic particles (SEP) offer the best method for determining solar elemental and isotopic composition, thereby greatly strengthening our knowledge of solar system composition. By comparing the composition at various flare sites along with solar wind and photospheric composition, it is possible to gain key insights into the physical mechanism which transports heavy ions into corona and then accelerates and transports into the interplanetary space.

The cosmic ray research is to study their origin, nucleosynthesis, particle acceleration and propagation, and their role in the dynamics of our galaxy. Isotopic ratios are the most genuine clues to the origin of GCR nuclei.

The GEOTAIL mission provides a good opportunity to study important problems in cosmic ray physics as well as solar physics. A broad range of processes occurring at solar/stellar atmosphere, and its interaction with the interplanetary/interstellar medium is investigated by the instrumental package in the High Energy Particle (HEP) experiment on the GEOTAIL mission. It is necessary for this research to observe not only major elements but their isotopic composition and rare elements. The study of their properties requires excellent charge-, mass- and energy-resolution, and large geometric factor. In order 
anisotropy (electron total)
(a) 14:11:30-14:12:00
(b) 14:12:00-14:12:30
(c) $14: 12: 30-14: 13: 00$
(d) $14: 13: 00-14: 13: 30$

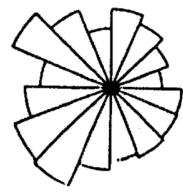

(e) 14:13:30-14:14:00

(1) 14:14:00-14:14:30


anisotropy (proton total)

(a) 14:11:30-14:12:00

(b) 14:12:00-14:12:30


(e)14:13:30-14:14:00

(f) 14:14:00-14:14:30
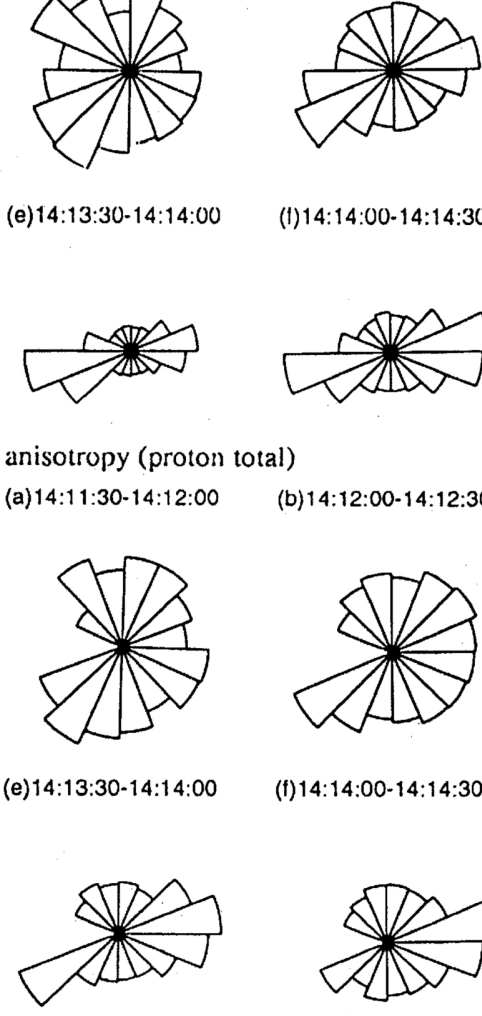

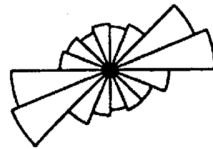

(g) 14:14:30-14:15:00

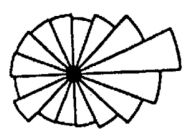

(c) 14:12:30-14:13:00

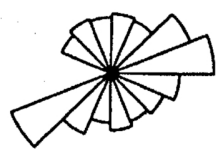

(g) 14:14:30-14:15:00

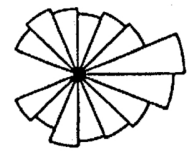

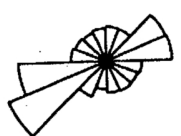

(h) 14:15:00-14:15:30

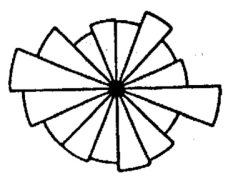

(d) 14:13:00-14:13:30

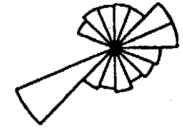

(h) 14:15:00-14:15:30

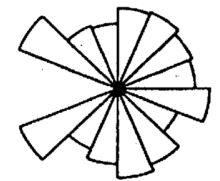

Fig. 8(b). Azimuthal anisotropy of particles detected by BD for several time intervals on January 31, 1993. Upper eight panels correspond to electrons; lower panels, protons. For the proton inisortropies, sectors affected by sunlight are omitted from the figure.

to meet those objectives, the instruments consisting of two telescope subsystems (the HI and MI) have been developed for the GEOTAIL.

\subsection{The isotope telescope system}

The instrumental package, consisting of two telescope subsystems and an electronics subsystem in the HEP experiment, has been developed in order to study the energetic particle phenomena. The telescopes are the High energy Isotope telescope $\mathrm{HI}$ and the Medium energy Isotope telescope MI. The MI includes a single MI-1 and two identical MI-2 units (MI-2A and MI-2B). By using the telescopes, the charge, mass and energy response have been optimized over a broad range of the energies.

\subsubsection{Characteristics of isotope telescopes}

The telescopes consist of all Si detectors utilizing the well-established $\Delta E \times E$ method for the isotope identification. They consist of mult-layers of Si detectors, of which top two layers are position-sensitive Si detectors (PSDs) in order to achieve a high mass resolution for isotopic analysis of incident particles and to increase the geometric factor of the telescope (Doke et al., 1987; Hasebe et al., 1988, 1992; Yanagimachi et al., 1988). Each PSD provides a two-dimensional position information using the chargedivision method. The PSDs also measure energy losses of the charged particles in those layers. The 


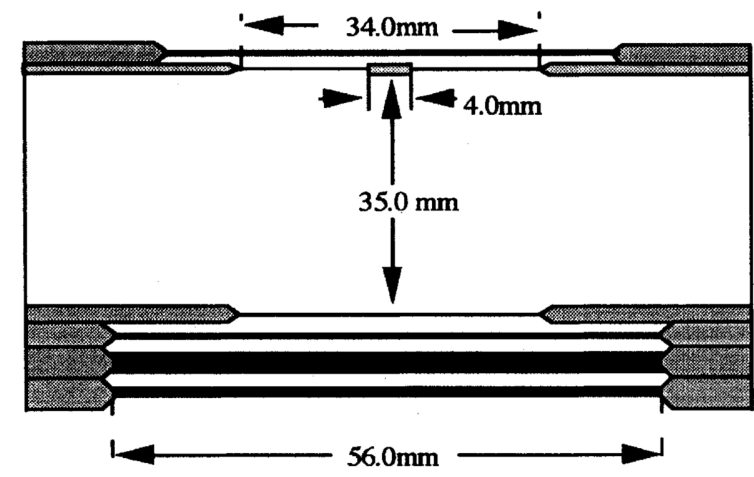

Medium energy Isotope telescope : MI 1

Window (0.1 $\mathrm{mmt}, 45 \mathrm{~mm}$, Poly Ether Imid)

PSD-1 ( $0.05 \mathrm{mmt}, 15 \mathrm{~mm}$, IISi )

PSD-2 ( $0.1 \mathrm{mmt}, 34 \mathrm{~mm}$, IISi)

$\mathrm{D} 1$ ( $0.4 \mathrm{mmt}, 56 \mathrm{mmb}, \mathrm{Si}(\mathrm{Li})$ )

D2 ( $2.0 \mathrm{mmt}, 56 \mathrm{~mm}$, $\mathrm{Si}(\mathrm{Li})$ )

D3 ( $1.0 \mathrm{mmt}, 56 \mathrm{~mm}, \mathrm{Si}(\mathrm{Li})$ )

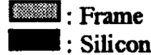

IISi : Ion-implanted Si-Detecor.

Window ( $0.1 \mathrm{mmt}, 70 \mathrm{~mm}$, Poly Ether Imid)

PSD-1 ( $0.1 \mathrm{mmt}, 28 \mathrm{~mm}$, IISi )

PSD-2 ( $0.2 \mathrm{mmt}, 56 \mathrm{~mm}$, IISi)

D1 ( $0.2 \mathrm{mmt}, 56 \mathrm{~mm}$, Si(Li))

$\mathrm{D} 2$ ( $0.4 \mathrm{mmt}, 62 \mathrm{~mm}, \mathrm{Si}(\mathrm{Li})$ )

$\mathrm{D} 3$ ( $2.0 \mathrm{mmt}, 62 \mathrm{~mm}, \mathrm{Si}(\mathrm{Li})$ )

D4 ( $1.0 \mathrm{mmt}, 62 \mathrm{~mm}$, Si(Li) )

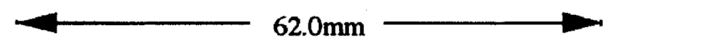

Medium energy Isotope telescopes : MI2 A\&B \%rame

IISi : Ion-implanted Si-Detector.



High energy Isotope telescope: HI
Window (0.1 mmt, 65mm , Poly Ether Imid) PSD-1 ( $0.5 \mathrm{mmt}, 62 \mathrm{~mm}$, IISi )

PSD-2 ( $0.5 \mathrm{mmt}, 62 \mathrm{~mm}$, IISi )

D1 ( $1 \mathrm{mmt}, 78 \mathrm{~mm}$. Si(Li))

$\mathrm{D} 2$ ( $2 \mathrm{mmt}, 114 \mathrm{~mm} \Phi, \mathrm{Si}(\mathrm{Li})$ )

D3 ( $3 \mathrm{mmt}, 114 \mathrm{~mm} \Phi, \mathrm{Si}(\mathrm{Li})$ )

D4 ( $3 \mathrm{mmt}, 114 \mathrm{~mm} \Phi, \mathrm{Si}(\mathrm{Li})$ )

D5 ( $3 \mathrm{mmt}, 114 \mathrm{~mm} \Phi, \mathrm{Si}(\mathrm{Li})$ )

D6 ( $2 \mathrm{mmt}, 114 \mathrm{~mm} \Phi, \mathrm{Si}(\mathrm{Li})$ )

5rin: : Frame

: Silicon

IISi : Ion-implanted Si-Detecor.

Fig. 9. Schematic drawing of the isotope telescopes in the high energy particle experiment on the GEOTAIL mission; the Medium energy Isotope telescope MI-1, the Medium energy Isotope telescopes MI-2A and MI-2B, the High energy Isotope telescope HI. 
Table 5. Characteristic of the isotope telescopes boarded on the GEOTAIL satellite.

\begin{tabular}{|c|c|c|c|}
\hline \multirow[t]{3}{*}{ Ions } & \multicolumn{3}{|c|}{ Energy range $(\mathrm{MeV} / \mathrm{n})$} \\
\hline & \multicolumn{2}{|c|}{ MI } & \multirow[t]{2}{*}{$\mathrm{HI}$} \\
\hline & MI-1 & MI-2A and B & \\
\hline $\mathrm{He}$ & $2.3-22$ & - & $10-55$ \\
\hline $\mathrm{C}$ & $4.0-46$ & $6.8-48$ & 21-104 \\
\hline $\mathrm{Ne}$ & $5.1-61$ & $9.0-64$ & $26-139$ \\
\hline $\mathrm{Ca}$ & - & $12-95$ & $37-207$ \\
\hline $\mathrm{Fe}$ & - & $13-104$ & $39-230$ \\
\hline$Z \leq 44$ & - & $13-136$ & - \\
\hline \multicolumn{4}{|l|}{ Charge range } \\
\hline for Elements & $2-10$ & $6-44$ & $2-28$ \\
\hline for Isotopes & $2-10$ & $6-26$ & $2-28$ \\
\hline $\begin{array}{l}\text { Mass resolution } \\
\text { (amu in fwhm) }\end{array}$ & $<0.5$ & $<0.5$ & $<0.5$ \\
\hline Geometric factor & $4.1 \mathrm{~cm}^{2} \mathrm{sr}$ & $26.7 \mathrm{~cm}^{2} \mathrm{sr} / \mathrm{each}$ & $43.2 \mathrm{~cm}^{2} \mathrm{sr}$ \\
\hline Field of view & $90^{\circ} \times 90^{\circ}$ & $120^{\circ} \times 120^{\circ}$ & $120^{\circ} \times 120^{\circ}$ \\
\hline Weight (kg) & \multicolumn{2}{|c|}{$4.72(\mathrm{MI}-1$ and $\mathrm{MI}-2)$} & 3.54 \\
\hline Power (W) & \multicolumn{2}{|c|}{1.69 (MI-1 and MI-2) } & 1.13 \\
\hline
\end{tabular}

following several layers are Si-detectors with various thickness for measuring energy losses or residual energy of stopping ions. General characteristics of the HI and MI telescopes are listed in Table 5.

The HI: A schematic drawing of the HI subsystem is shown in Fig. 9. The nominal physical characteristics of the Si detectors used in the $\mathrm{HI}$ are also shown in the figure. The telescope consists of large PSDs and co-axially arrayed Li-drifted Si detectors. In order to shield the sun light and to absorb the low energy proton and helium ions, a $0.050 \mathrm{~mm}$ thick window made of PolyEtherImid (PEI) is placed in front of the Si detector stack. The energy range covered by the $\mathrm{HI}$ is from 10 to $230 \mathrm{MeV} / \mathrm{n}$, depending on the nuclear charge. The HI has very large geometric factor, $43-45 \mathrm{~cm}^{2} \mathrm{sr}$, that we can measure heavy isotopes up to $\mathrm{Fe} / \mathrm{Ni}$ with relatively high energies in GCR and large solar flare events.

The MI: The MI subsystem consists of the MI-1, MI-2A and MI-2B units. The detector configuration is similar to that of the HI. The top layer (PSD1), however, is different from the HI in the structure of the electrodes. The PSD1 has four position-sensitive regions. Schematic drawings of the MI-1 and the MI2 telescopes are also shown in Fig. 9. The MI-2A/B has such an exceptionally large geometric factor, 26.7 $\mathrm{cm}^{2} \mathrm{sr} / \mathrm{each}$, that we intensively observe heavy isotopes up to $\mathrm{Fe} / \mathrm{Ni}$ nuclei with relatively low energies from 7 to $136 \mathrm{MeV} / \mathrm{n}$ in small and large solar flare events. The MI-1 telescope measures the low-Z isotopes of He-Ne with low energies from 3 to $61 \mathrm{MeV} / \mathrm{n}$ in flare particle events and anomalous cosmic rays.

The telescope subsystems, the HI and the MI, are thermally insulated by $12 \mathrm{~mm}$ thick spacer from the spacecraft platform and enclosed by multilayer insulation blankets except for the front surface open to the space. In the front surface, optical solar reflector of silver deposited PolyEtherImid (PEI) is attached to radiate heat to the space.

The electronics subsystem, HEP-E; includes a pulse height analyzer unit and a data processing unit (DPU). Preamplifier signals in the HI and MI subsystems are fed to the main shaping amplifiers in the HEP-E. The peaks of the amplified signals are detected and retained by peak-detection and sample-hold circuitry. The information of particle trajectories and energy losses is provided by pulse height analysis (PHA) of 16-bit ADCs.

The DPU including two CPU(Z80) systems has a function of data processing and transmission, 
command interpretation, execution and so on. The in-flight calibration checks, on command, the performance of the instrument, such as amplifier gains and linearity of the ADCs by delivering a sequence of pulses to all preamplifiers.

\subsubsection{Performance of isotope telescopes}

The characteristics of the telescopes and the individual Si detectors were examined by using heavy ion beams from the LBL Bevalac and RIKEN Ring-Cyclotron accelerators (Hasebe et al., 1992, 1993a, 1993b).

By irradiating the ion beams with the monochromatic energy, the non-uniformity of the detector thickness was measured from the distribution of the energies deposited in the detectors, using the position information from PSDs. The non-uniformity of the thickness obtained for the $\mathrm{Si}(\mathrm{Li})$ detectors is $<1.1 \%$ in fwhm for the whole area for the $\mathrm{HI},<0.54 \%$ for the MI- 1 , and $<0.98 \%$ for the MI-2A and MI-2B. Thus the thickness uniformity obtained is almost satisfactory to identify isotopes up to Fe.

The position performance of the PSDs in the HI and MI was also measured by using the parallel $\mathrm{O}$ and Fe beams with the monochromatic energies (Hasebe et al., 1992). Position resolutions for the $x$ - and $y$-direction were 1.1 and $0.99 \mathrm{~mm}$ in fwhm, respectively, when energies of 242 and $237 \mathrm{MeV}$ were deposited in the PSDs of the HI. The resolution depends on the energy deposition in the detectors. Similar results were obtained for the MI-1, and MI-2A/B.

To calibrate the telescope response of the telescopes to various heavy ions in space, ion beams produced by ${ }^{56} \mathrm{Fe}$ beam with a polyethylene target were irradiated to the telescopes (Hasebe et al., 1993a, 1993b). The response of the HI and the MI was investigated with the Bevalac accelerator at LBL and the Ring-cyclotron at RIKEN, respectively. Elements and their isotopes from He to Fe were clearly resolved as the results. The good mass resolution thus obtained in the beam experiment are 0.38-0.47 amu in fwhm for $\mathrm{He}$ to $\mathrm{Fe}$, which lead to an excellent identification of particles in SEP and GCR.

\subsection{Initial results of HI/MI spectrometers}

The GEOTAIL satellite was launched in July, 1992 and now in full operation moving around in the scheduled orbit. Scientific payload on the GEOTAIL was switched-on in September, 1992 and then functions of the telescopes were checked. The observation of energetic heavy ions has been made by the MIs and the HI since then. The intermittent observation had been unfortunately made until the end of June, 1993 because of the CPU malfunction which was solved by the software disposal which made the continuous observation since July 1993. Figure 10 shows the observational result of the charge histogram

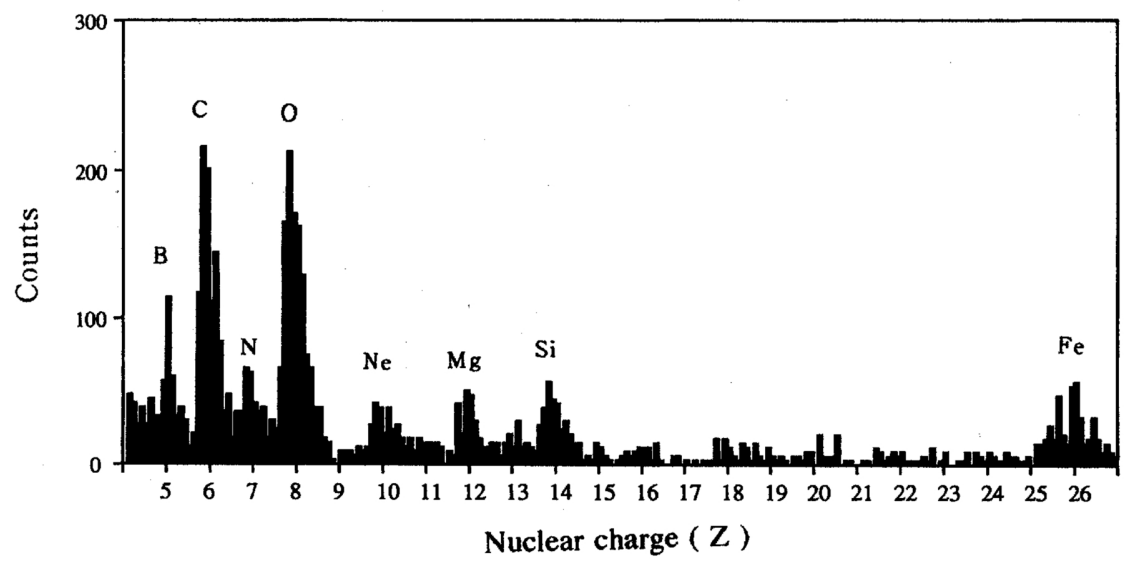

Fig. 10. The histogram of galactic cosmic rays from the HI telescope in the $Z=5-26$ charge range. Abundance elements in GCR are clearly resolved. Particle event data in the quiet time of 1742 hours in the period from Sept. 1, 1992 to Aug. 31,1993 are taken in the analysis. 
$(Z \geq 5)$ for the first 1472 hours observed by using the HI telescope. As seen from this figure, major elements such as $\mathrm{C}, \mathrm{N}, \mathrm{O}, \mathrm{Ne}, \mathrm{Mg}, \mathrm{Si}$ and $\mathrm{Fe}$, which stopped in the telescope are obviously resolved. The result shown in the figure is roughly calibrated on the energy and positions of PSDs and $\mathrm{Si}(\mathrm{Li})$. To observe the odd nuclei such as $\mathrm{F}, \mathrm{Na}, \mathrm{P}, \mathrm{Cl}, \mathrm{K}, \mathrm{Sc}, \mathrm{V}, \mathrm{Mn}$ and $\mathrm{Co}$ with good statistics, a longer period of observation is required.

The isotope telescopes have very large geometric factors of $44 \mathrm{~cm}^{2} \mathrm{sr}$ for the HI, and of $58 \mathrm{~cm}^{2} \mathrm{sr}$ in total for the MI, covering the broad energy range of 3.0-230 MeV/n. The telescopes so far aboard the IMP-8/9 (Garcia-Munoz et al., 1977), ISEE-3 (Althouse et al., 1978; Greiner et al., 1978) and Voyager1/2 (von Rosenvinge et al., 1978; Stone et al., 1977; Stilwell et al., 1979) spacecraft launched in the 1970s, have the geometric factors of about a few $\mathrm{cm}^{2} \mathrm{sr}$ or less. The GEOTAIL observes energetic ions in the period from the declining phase to the minimum of solar activity. Particle observation during four years of mission life will make possible the precise determination of the chemical abundance, which will make a great progress in the study.

Observation of low energy cosmic ray particles in the energies from 5 to $50 \mathrm{MeV} / \mathrm{n}$ by the HEP instrument $\mathrm{MI}$ and $\mathrm{HI}$ shows a remarkable enhancement of anomalous cosmic ray (ACR) $\mathrm{N}$ and $\mathrm{O}$ in the period of 9.1992-8.1993 before approaching to the solar minimum in the solar cycle 22. Figure 11 shows the energy spectra for $\mathrm{N}$ and $\mathrm{O}$ for a quiet period between September 1992 and August 1993. The quiettime intervals were selected such that the $3-5 \mathrm{MeV} / \mathrm{n}$ He intensity was typically less than 1.3 particles $\left(\mathrm{cm}^{2}\right.$ $\sec \mathrm{sr} \mathrm{MeV} / \mathrm{n})^{-1}$. The $\mathrm{N}$ and $\mathrm{O}$ fluxes in the energy region around $\sim 50 \mathrm{MeV} / \mathrm{n}$ are consistent with typical galactic cosmic ray component. The $\mathrm{N}$ and $\mathrm{O}$ fluxes have rapidly increased at energies below $40 \mathrm{MeV} /$ $\mathrm{n}$, although this prominent enhancement of the ACR nuclei at $1 \mathrm{AU}$ was not expected just after the solar maximum on the basis of the ACR observations in the previous cycle. The intensity levels of the observed ACRs agree with that from the SAMPEX obtained during late 1992 to early 1993 (Mewaldt et al., 1993). The intensity of the ACR component is still increasing toward the solar minimum. Temporal variation of the ACR component roughly correlates with the neutron monitor counting rate. The increasing rate of the ACR intensity in this solar cycle, however, is a few times larger than that of the neutron level.

Energy spectra of He and $\mathrm{O}$ for the 1993 March 4-6 solar flare event are presented in Fig. 12. He and $\mathrm{O}$ fluxes at energies below $45 \mathrm{MeV} / \mathrm{n}$ measured by the MI-1 shown by open circle and triangle are from

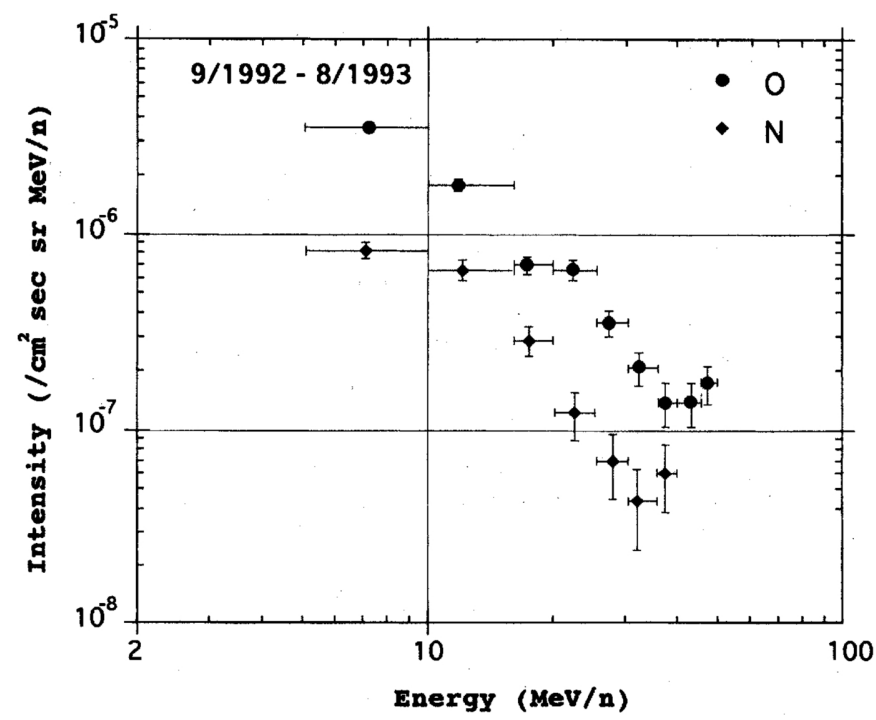

Fig. 11. Differential energy spectra of anomalous nitrogen and oxygen during quiet time from September 1992 to August 1993 from the GEOTAIL satellite. 




Fig. 12. Differential energy spectra of $\mathrm{He}$ and $\mathrm{O}$ taken from the increasing phase of 3 hours from 14:00 to 17:00 UT on March 4 in 1993, and the declining phase from 13:22 on March 5 to 24:00 UT on March 6 in 1993. Those spectral indices differ from each other.

the increasing phase of particle event for 3 hours from 14:00 to 17:00 UT of 1993 March 4. The energy spectra shown as solid symbols are from the declining phase from 13:22 March 5 to 24:00 March 6, 1993. The rapid increase of the counting rates on March 4 seems to be caused by small flare starting on 1993 March 4 at 1016 UT. The optical and X-ray class of the flare located at the region 7435 is $1 \mathrm{~N}$ and M1.0, respectively. The energy spectra of $\mathrm{He}$ for this event is steeper than that of $\mathrm{O}$. As seen from the spectra, their spectral indices are different from one phase to another. And their spectral indices of the $\mathrm{He}$ and $\mathrm{O}$ in the declining phase of the event are steeper than ones in the increasing phase. Small SEP events like this event observable in the low energy region occur frequently even in the declining phase of solar activity.

We are equally grateful for the exceptional management practiced by Prof. T. Mukai and the GEOTAIL project manager team: Prof. K. Uesugi and Prof. I. Nakatani at ISAS/Tokyo. We acknowledge with pleasure the excellent collaboration sense rather than by asking for immense loads of formal documentation.

The work at the MPAe and at IDA was supported by a grant from the Deutche Agentur für Raumfahrtangelelgenheiten (DARA), the University of Bergen received support from the Norwegian Research Council for Science and the Humanities.

We wish to thank the LBL and its staff, the RIKEN and its staff, the INS and its staff for their generous support in providing heavy ion beams for the testing and calibration of the instrument.

We are grateful for the cooperation of the staff of MEC for the fabrication and various tests of the instrument, NEC for the various tests, and Hamamatsu Photonics for the detector development.

The Editor would like to thank M. Kojima and one other referee for their assistance in evaluating this paper.

\section{REFERENCES}

Althouse, W. E., A. C. Cummings, T. L. Garrard, R. A. Mewaldt, E. C. Stone, and R. E. Vogt, A cosmic ray isotope spectrometer, IEEE, Trans., GE-16, No. 3, 204-207, 1978.

Berger, M. J., S. M. Seltzer, S. E. Chappell, J. C. Humphreys, and J. W. Morz, Table of response functions for silicon detectors, Nat. Bur. Stand. (U.S.), Techn. Note 89 (Aug.), 1969. 
Daly, P. W., T. R. Sanderson, and K. P. Wenzel, Survey of energetic $(E>35 \mathrm{keV})$ ion anisotropies in the deep geomagnetic tail, J. Geophys. Res., 89, 10733-10739, 1984.

Doke, T., J. Kikuchi, H. Yamaguchi, S. Yamaguchi, and K. Yamamura, A new two-dimensional position sensitive detector with a good linear response, Nucl. Instrum. Method, A261, 605-609, 1987.

Doke, T., J. Kikuchi, K. Nishijima, N. Hasebe, H. Murakami, A. Nakamoto, T. Yanagimachi, K. Ito, T. Kohno, K. Nagata, B. Wilken, K. Maezawa, T. Terasawa, S. Yanagita, and A. Nishida, High energy particle experiments for the Geotail mission, ISAS report SP No. 10, 1989.

Doke, T., J. Kikuchi, K. Nishijima, N. Hasebe, H. Murakami, A. Nakamoto, T. Yanagimachi, K. Ito, T. Kohno, K. Nagata, B. Wilken, K. Maezawa, T. Terasawa, S. Yanagita, and A. Nishida, High energy particle experiment in Geotail mission, 21 st ICRC Conference Papers, 7, 272-275, 1990a.

Doke, T., J. Kikuchi, N. Hasebe, H. Murakami, A. Nakamoto, T. Yanagimachi, K. Ito, T. Kohno, K. Nagata, B. Wilken, K. Maezawa, T. Terasawa, S. Yanagita, and A. Nishida, High energy particle experiment (HEP), GEOTAIL INTERIM REPORT, ISAS SES-TD-90-021SY, 65-89, 1990b.

Doke, T., J. Kikuchi, T. Hayashi, T. Kashiwagi, T. Shino, K. Itsumi, N. Hasebe, H. Moriya, K. Fujiki, T. Yanagimachi, H. Murakami, A. Nakamoto, K. Nagata, and T. Kohno, The Geotail energetic isotope telescopes, Meas. Sci. Technol., 1994 (to be published).

Garcia-Munoz, M., G. M. Mason, and J. A. Simpson, The age of the galactic cosmic rays derived from the abundance of ${ }^{10} \mathrm{Be}$, Astrophys. J., 217, 859, 1977.

Greiner, D. E., F. S. Bieser, and H. H. Heckman, ISEE-C HKH high energy cosmic rays, IEEE Trans., GE-16, No. 3, 163-165, 1978.

Hasebe, N., Y. Ezawa, H. Yoshii, and T. Yanagimachi, Charge waveform of a new two-dimensional position-sensitive silicon detector, Jpn. J. Appl. Phys., 27, 816-820, 1988.

Hasebe, N., T. Doke, T. Kashiwagi, S. Kataoka, J. Kikuchi, H. Moriya, K. Nishijima, and H. J. Crawford, Position linearity and resolution of large position-sensitive silicon detector with a highly uniform thickness, Jpn. J. Appl. Phys., 31, 1191-1193, 1992.

Hasebe, N., H. Moriya, T. Doke, J. Kikuchi, T. Kashiwagi, S. Kataoka, T. Shino, K. Itsumi, T. Hayashi, T. Yanagimachi, A. Nakamoto, H. Murakami, K. Nagata, H. J. Crawford, J. M. Engelarge, I. Flores, and L. Greiner, Improvement of mass resolution of cosmic ray nuclei using a $\Delta E \times E$ Si detector telescope, Nucl. Instr. Meth., A325, 335-342, $1993 \mathrm{a}$.

Hasebe, N., H. Moriya, K. Fujiki, T. Doke, J. Kikuchi, T. Hayashi, T. Kashiwagi, T. Shino, K. Itsumi, T. Yanagimachi, H. Murakami, A. Nakamoto, K. Nagata, and T. Kohno, The GEOTAIL energetic isotope telescopes, J. Phys., 1993b (to be submitted).

Mewaldt, R. A., A. C. Cummings, J. R. Cummings, E. C. Stone, and T. T. von Rosenvinge, The return of the anomalous component to $1 \mathrm{AU}$ in 1992, Proc. 23rd ICRC (Calgary), 3, 404-407, 1993.

Scholer, M., D. Hovestadt, B. Klecker, G. Gloeckler, and F. M. Ipavich, Average flow between $70 \operatorname{Re}$ and $220 \mathrm{Re}$ in the geomagnetic tail, Geophys. Res. Lett., 11, 343-346, 1984.

Stilwell, D. E., W. D. Davis, R. M. Joyce, F. B. McDonald, J. H. Trainor, W. E. Althouse, A. C. Cummings, T. L. Garrard, E. C. Stone, and R. E. Vogt, The Voyager cosmic ray experiment, IEEE Trans., NS-26, No. 1, 513-520, 1979.

Stone, E. C., R. E. Vogt, F. B. McDonald, B. J. Teegarden, J. H. Trainor, J. R. Jokipii, and W. R. Webber, Cosmic ray investigation for the Voyager missions; energetic particle studies in the outer heliosphere and beyond, Space Sci. Rev., 21, 355-376, 1977.

von Rosenvinge, T. T., F. B. McDonald, J. H. Trainor, M. A. I. Van Hollebeke, and L. A. Fisk, The medium energy cosmic ray experiment for ISEE-C, IEEE Trans., GE-16, No. 3, 208-212, 1978.

Wilken, B., K. Nagata, T. Doke, F. Gliem, W. Guettler, N. Hasebe, S. Livi, H. Murakami, R. Rathje, H. Sommer, S. Ullaland, L. Ersland, A. Sandvand, W. Weiss, and T. Yanagimachi, The energetic particle spectrometer HEP-LD onboard the Japanese tailprobe GEOTAIL, Meas. Sci. Technol., 1994 (to be published).

Yanagimachi, T., T. Doke, N. Hasebe, T. Imai, T. Kashiwagi, J. Kikuchi, T. Kohno, W. P. Liu, K. Munakata, T. Motobayashi, H. Murakami, K. Nagata, A. Nakamoto, and H. Yamaguchi, New two-dimensional position sensitive silicon detector with good position linearity and resolution, Nucl. Instr. Meth., A275, 307-317, 1988. 